F. Azarpanah, Department of Mathematics, Chamran University, Ahvaz, Iran. e-mail: azarpan@www.dci.co.ir

\title{
ALGEBRAIC PROPERTIES OF SOME COMPACT SPACES
}

\begin{abstract}
Almost discrete spaces and in particular, the one-point compactifications of discrete spaces are algebraically characterized. This algebraic characterization is then used to show that whenever $C(X) \approx C(Y)$ and $X$ is the one-point compactification of a discrete space, then $Y$ is too. Some equivalent algebraic properties of almost locally compact spaces and nowhere compact spaces are studied. Using these properties we show that every completely regular space can be decomposed into two disjoint subspaces, where one is an open almost locally compact space and the other is a nowhere compact space. Finally, we will show that $X$ is Lindelöf if and only if every strongly divisible ideal in $C(X)$ is fixed.
\end{abstract}

\section{Introduction}

A nonzero ideal in a commutative ring $R$ is called essential if it intersects every nonzero ideal nontrivially. The intersection of all essential ideals in any commutative ring $R$, or the sum of all minimal ideals of $R$ is the socle of $R$, (see [6]). $C(X)$ denotes the ring of all continuous real valued functions on a completely regular Hausdorff space $X$. For any $f \in C(X), Z(f)$ denotes the set of zeros of $f$ and for every ideal $I$ in $C(X)$, if $\cap Z[I]=\cap_{f \in I} Z(f)$ is nonempty, $I$ is called fixed, else free. For every $p \in X$, the ideal $O_{p}$ is defined to be the set $\left\{f \in C(X): p \in \operatorname{int}_{X} Z(f)\right\}$, where $\operatorname{int}_{X}$ denotes interior in $X$. Fixed maximal ideals of $C(X)$ are of the form $M_{p}=\{f \in C(X): p \in Z(f)\}$, for $p \in X$. More generally, $M^{p}=\left\{f \in C(X): p \in \mathrm{cl}_{\beta X} Z(f)\right\}$, where $\beta X$ is the Stone-Čech compactification of $X$ and $\operatorname{cl}_{\beta X}$ denotes closure in $\beta X$, is a maximal ideal, fixed or free in $C(X)$, (see [5]). Related to these are the ideals

\footnotetext{
Key Words: essential ideal, strongly divisible ideal, almost locally compact, nowhere compact, Lindelöf and almost discrete space

Mathematical Reviews subject classification: $54 \mathrm{C} 40$

Received by the editors December 1, 1998

* The author is partially supported by the Institute of Theoretical Physics and Mathematics (IPM)
} 
$O^{p}=\left\{f \in C(X): p \in \operatorname{int}_{\beta X} \operatorname{cl}_{\beta X} Z(f)\right\}, p \in \beta X$. If $p \in X$, then $M^{p}=M_{p}$ and $O^{p}=O_{p}$. For every subset $B$ of a commutative ring $R, \operatorname{Ann}(B)=\{a \in$ $R: a B=(0)\}$, in particular, for every $b \in R, \operatorname{Ann}(b)=\{a \in R: a b=0\}$.

For every $f \in C(X)$, the support of $f$ is the closure of $X \backslash Z(f)$ and the ideal $C_{K}(X)$ denotes the family of all functions in $C(X)$ having compact support. In $7 \mathrm{E}$ of [5] it is shown, that $C_{K}(X)$ is actually the intersection of all free ideals in $C(X)$. A function $f \in C(X)$ is said to vanish at infinity if the set $\left\{x \in X:|f(x)| \geq \frac{1}{n}\right\}$ is compact for every $n \in \mathbb{N}$. The ideal $C_{\infty}(X)$ is the family of all functions in $C(X)$ which vanish at infinity. $C_{\infty}(X)$ is the intersection of all free maximal ideals in $C^{*}(X)$, the ring of all continuous bounded real valued functions on $X$, (see $7 \mathrm{E},[5]$ ).

In this paper $X$ denotes a completely regular Hausdorff space and the reader is referred to [5] for undefined terms and notations.

The following propositions which topologically characterize essential ideals and the socle of $C(X)$ are proved in [1] and [8] respectively.

Proposition 1.1. A nonzero ideal $E$ in $C(X)$ is essential if and only if $\cap Z[E]$ is nowhere dense in $X$.

Proposition 1.2. The socle $C_{F}(X)$ of $C(X)$ is a z-ideal consisting of all functions vanishing everywhere except on a finite number of points of $X$.

\section{One-Point Compactification and Almost Discrete Spaces}

In this section we will prove that $X$ is the one-point compactification of a discrete space if and only if for some $x \in X$, the ideal $O_{x}$ and the socle of $C(X)$ coincide. Using this fact, we will show that the socle of $C(X)$ is never a prime ideal. More generally, if $A \subseteq X$ is closed, then $O_{A}=\cap_{x \in A} O_{x} \subseteq C_{F}(X)$ if and only if every open set containing $A$ has a finite complement. We also prove a similar result for the one-point compactification of any locally compact space. Almost discrete spaces (The spaces in which the set of isolated points is dense.) are algebraically characterized and the last result of this section shows that the topological structure of the one-point compactification of a discrete space is invariant under ring isomorphisms.

Proposition 2.1. The infinite space $X$ is the one-point compactification of a discrete space if and only if for some $x \in X, O_{x} \subseteq C_{F}(X)$ (or equivalently, $\left.O_{x}=C_{F}(X)\right)$.

Proof. If $O_{x} \subseteq C_{F}(X)$, then $x$ can not be an isolated point, for otherwise there is $f \in C(X)$ such that $f(x)=0$ and $f(X \backslash\{x\})=\{1\}$. Then $f \in$ $O_{x} \subseteq C_{F}(X)$ implies that $X \backslash Z(f)=X \backslash\{x\}$ is finite, a contradiction. 
Now we will show that $x$ is the only nonisolated point of $X$. Suppose that $y \neq x$ is another nonisolated point of $X$. By Theorem 1.15 in [5], there exist $f, g \in C(X)$ such that $x \in \operatorname{int} Z(f), y \in \operatorname{int} Z(g)$ and $Z(f) \cap Z(g)=\phi$. This implies that $f \in O_{x} \subseteq C_{F}(X)$ and $X \backslash Z(f)$ contains $Z(g)$. But, since $Z(g)$ is a neighborhood of the nonisolated point $y$, it is an infinite set which implies that $f \notin C_{F}(X)$, a contradiction. Now let $G$ be an open set which contains $x$. Again by Theorem 1.15 in [5], there exists $h \in C(X)$ such that $x \in \operatorname{int} Z(h) \subseteq Z(h) \subseteq G$. So $h \in O_{x} \subseteq C_{F}(X)$ and Proposition 1.2 implies that $X \backslash Z(h)$ and hence $X \backslash G$ is finite. Therefore every open set containing $x$ has a compact (finite) complement; i.e., $X$ is the one-point compactification of a discrete space.

Conversely, let $X=Y \cup\{x\}$ be the one-point compactification of a discrete space $Y$. We will show that $O_{x}=C_{F}(X)$. If $f \in O_{x}$, then int $Z(f)$ is an open set containing $x$, which must have a compact complement in $Y$. This implies that $X \backslash Z(f)$ is finite; i.e., $f \in C_{F}(X)$. If $f \in C_{F}(X)$, then $X \backslash Z(f)$ is finite and hence $x \notin X \backslash Z(f)$, for $x$ is not an isolated point. Therefore $x \in Z(f)=\operatorname{int} Z(f)$, implies that $f \in O_{x}$.

Remark 2.2. For a generalization of Proposition 2.1, if $A$ is a closed set in $X$, then $O_{A} \subseteq C_{F}(X)$ if and only if every open set in X containing A has a finite complement. To see this, let $O_{A} \subseteq C_{F}(X)$ and $G$ be an open set with $A \subseteq G$. If $x \in X \backslash G$, then $A$ and $x$ are completely separated and by Theorem 1.15 in [5], they are contained in two disjoint zero-set neighborhoods say $Z(f)$ and $Z(g)$. If $A \subseteq \operatorname{int} Z(f)$ and $x \in \operatorname{int} Z(g)$, then $f \in O_{A} \subseteq C_{F}(X)$. Now if $x$ is not an isolated point, then the open set $X \backslash Z(f)$ which contains $x$ must be infinite which contradicts $f \in C_{F}(X)$. Therefore $X \backslash G$ is an open-closed set. Now define $h \in C(X)$ such that $h(G)=\{0\}$ and $h(X \backslash G)=\{1\}$. Then $h \in O_{A} \subseteq C_{F}(X)$ implies that $X \backslash Z(h)=X \backslash G$ is finite.

Conversely, let every open set containing $A$ have a finite complement and $f \in O_{A}$. Then $A \subseteq \operatorname{int} Z(f)$. Hence $X \backslash \operatorname{int} Z(f)$ is finite by our hypothesis and then $X \backslash Z(f)$ is also finite or $f \in C_{F}(X)$; i.e., $O_{A} \subseteq C_{F}(X)$.

Remark 2.3. Whenever $Y$ is a locally compact space and $X=Y \cup\{x\}$, then $O_{x} \subseteq C_{K}(X)$, if and only if $X$ is the one-point compactification of the space $Y$. To prove this, let $O_{x} \subseteq C_{K}(X)$ and $G$ be an open set which contains $x$. Then by Theorem 1.15 in [5], there exists $f \in C(X)$ such that $x \in \operatorname{int} Z(f) \subseteq Z(f) \subseteq G$. Therefore $f \in O_{x} \subseteq C_{K}(X)$, hence $\operatorname{cl}(X \backslash Z(f))$ is compact. But $X \backslash G \subseteq \operatorname{cl}(X \backslash Z(f))$ implies that $X \backslash G$ is also compact. Conversely, if $X=Y \cup\{x\}$ is the one-point compactification of $Y$ and $f \in O_{x}$, then $x \in \operatorname{int} Z(f)$ implies that $X \backslash \operatorname{int} Z(f)=\operatorname{cl}(X \backslash Z(f))$ is compact; i.e., $f \in C_{K}(X)$. Hence $O_{x} \subseteq C_{K}(X)$. 
It is well-known that if $W$ is the space of countable ordinals, then the lone free maximal ideal $M^{w_{1}}$ in $C(W)$ is precisely $C_{K}(W)$, (see [5], 8.19). The following proposition characterizes topological spaces $X$ for which $C_{K}(X)$ is a prime ideal.

Proposition 2.4. $C_{K}(X)$ is a prime ideal if and only if $X$ is noncompact locally compact and for any two disjoint cozero-sets, one has compact closure.

Proof. Let $C_{K}(X)$ be a prime ideal $P$. Then $P$ is not fixed, for in this case $O_{x} \subseteq C_{K}(X)$ for some $x \in X$ and by Remark 2.3, $X$ must be compact space. Hence $C_{K}(X)=C(X)$ by $4 \mathrm{D}$ in [5], a contradiction. Then $C_{K}(X)=P$ is free and again by $4 \mathrm{D}$ in [5], $X$ is a noncompact locally compact space. Now if $(X \backslash Z(f)) \cap(X \backslash Z(g))=\phi$ for some $f, g \in C(X)$, then $f g=0 \in C_{K}(X)=P$ implies that $f \in C_{K}(X)$ or $g \in C_{K}(X)$; i.e., either $\operatorname{cl}(X \backslash Z(f))$ or $\operatorname{cl}(X \backslash Z(g))$ is compact. Conversely, if $X$ is noncompact locally compact, then $C_{K}(X)$ is free by $4 \mathrm{D}$ in [5]. Now if $f g=0$, then $(X \backslash Z(f)) \cap(X \backslash Z(g))=\phi$ and by our hypothesis, either $\operatorname{cl}(X \backslash Z(f))$ or $\operatorname{cl}(X \backslash Z(g))$ is compact; i.e., either $f \in C_{K}(X)$ or $g \in C_{K}(X)$. Since $C_{K}(X)$ is a z-ideal, by [5], 2.9, $C_{K}(X)$ is a prime ideal.

This result raises a similar question for $C_{F}(X)$. When is $C_{F}(X)$ a prime ideal? In the following proposition we will answer this question.

Proposition 2.5. $C_{F}(X)$ is never a prime ideal.

Proof. Let $C_{F}(X)$ be a prime ideal. Suppose that $C_{F}(X)$ is free. Then $X$ must be a discrete space. If we separate $X$ into two disjoint infinite sets $A$ and $B(A \cup B=X)$ and put $Z(f)=A$ and $Z(g)=B$, then $f g=0$, but $f \notin C_{F}(X)$, $g \notin C_{F}(X)$, a contradiction. Therefore we may suppose that $C_{F}(X)$ is a fixed prime ideal. Then $O_{x} \subseteq C_{F}(X)$ for some $x \in X$ and by Proposition 2.1, $X$ is the one-point compactification of a discrete space with additional point $x$. Now we consider two disjoint countable infinite subset $C=\left\{x_{1}, x_{2}, \ldots\right\}$ and $D=\left\{y_{1}, y_{2}, \ldots\right\}$ of $X \backslash\{x\}$. Define $f_{n} \in C(X)$ such that $f_{n}\left(x_{n}\right)=1$ and $f_{n}\left(X \backslash\left\{x_{n}\right\}\right)=\{0\}$. Since $f(x)=\sum_{n=1}^{\infty} \frac{1}{2^{n}} f_{n}(x)$ converges uniformly on $X, f \in C(X)$ and $Z(f)=X \backslash C$. Similarly, we define a function $g \in C(X)$ such that $Z(g)=X \backslash D$. Clearly $f g=0$, but $f \notin C_{F}(X), g \notin C_{F}(X)$, a contradiction. Therefore $C_{F}(X)$ can not be a prime ideal.

To prove the last result of this section, we need the following lemma and proposition.

Lemma 2.6. (i). If $B \subseteq C(X)$ is a multiplicatively closed (m.c.) set, then

$$
\sum_{f \in B} \operatorname{Ann}(f)=\bigcup_{f \in B} \operatorname{Ann}(f) .
$$


(ii). For every $p \in \beta X, O^{p}=\sum_{f \notin M^{p}} \operatorname{Ann}(f)=\bigcup_{f \notin M^{p}} \operatorname{Ann}(f)$.

Proof. (i). It is clear that $\bigcup_{f \in B} \operatorname{Ann}(f) \subseteq \sum_{f \in B} \operatorname{Ann}(f)$. Conversely, let $g \in \sum_{f \in B} \operatorname{Ann}(f)$. Then $g=g_{1}+g_{2}+\cdots+g_{k}$, where $g_{i} \in \operatorname{Ann}\left(f_{i}\right), f_{i} \in B$, $i=1,2, \ldots k$. Since $B$ is a m.c. set, $f=\prod_{i=1}^{k} f_{i} \in B$ and hence $f g=0$ implies that $g \in \operatorname{Ann}(f)$; i.e., $g \in \bigcup_{f \in B} \operatorname{Ann}(f)$. Then $\sum_{f \in B} \operatorname{Ann}(f) \subseteq$ $\bigcup_{f \in B} \operatorname{Ann}(f)$.

(ii). By part $(i), \sum_{f \notin M^{p}} \operatorname{Ann}(f)=\bigcup_{f \notin M^{p}} \operatorname{Ann}(f)$, for $C(X) \backslash M^{p}$ is a m.c. set. The proof that $O^{p}=\bigcup_{f \notin M^{p}} A n n(f)$ is immediate by 7.12(b) in [5].

Proposition 2.7. $\operatorname{Ann}\left(C_{F}(X)\right)$ is generated by an idempotent if and only if $X$ is the union of two disjoint open subspaces $A$ and $N$, where $A$ is almost discrete and $N$ is dense in itself. In particular, $\operatorname{Ann}\left(C_{F}(X)\right)=(0)$ if and only if $X$ is almost discrete.

Proof. First suppose that $\operatorname{Ann}\left(C_{F}(X)\right)=(e)$, where $e$ is an idempotent in $C(X)$. Let $H$ be the set of isolated points of $X$. Since $e C_{F}(X)=(0)$, $H \subseteq Z(e)$ implies that $\operatorname{cl} H \subseteq Z(e)$. Now consider $x \in Z(e)$ but $x \notin \operatorname{cl} H$. Then by complete regularity of $X$, there exists $g \in C(X)$ such that $g(\operatorname{cl} H)=$ $\{0\}$ and $g(x)=1$. Clearly, $g \in \operatorname{Ann}\left(C_{F}(X)\right)$, but $g \notin(e)$, a contradiction. Hence $\operatorname{cl} H=Z(e)$ and since $e$ is an idempotent, $\mathrm{cl} H$ is open. Now $X=$ $\operatorname{cl} H \cup(X \backslash \operatorname{cl} H), \operatorname{cl} H$ and $(X \backslash \operatorname{cl} H)$ are disjoint open subspaces of $X, \operatorname{cl} H$ is almost discrete and $(X \backslash \mathrm{cl} H)$ is dense in itself.

Conversely, let $X=A \cup N$, where $A$ and $N$ are two disjoint open subspaces, $A$ almost discrete and $N$ dense in itself. If we define $e \in C(X)$ such that $e(A)=\{0\}$ and $e(N)=\{1\}$, then $e$ is an idempotent in $C(X)$. We will show that $\operatorname{Ann}\left(C_{F}(X)\right)=(e)$. Clearly $e \in \operatorname{Ann}\left(C_{F}(X)\right)$, for if $f \in C_{F}(X)$, then $X \backslash Z(f)$ is a finite open set and hence its members are isolated points; i.e., $X \backslash Z(f) \subseteq A=Z(e)$, or $f e=0$. Then $(e) \subseteq \operatorname{Ann}\left(C_{F}(X)\right)$. Now if $f \in$ $\operatorname{Ann}\left(C_{F}(X)\right)$, then $Z(e)=A \subseteq Z(f)$. Since $Z(e)$ is open, $Z(e) \subseteq \operatorname{int} Z(f)$ and by $1 \mathrm{D}$ in [5], $f$ is a multiple of $e$; i.e., $f \in(e)$ and hence $\operatorname{Ann}\left(C_{F}(X)\right) \subseteq(e)$.

Corollary 2.8. (i). If $X$ is an almost discrete space and $C(X)$ is isomorphic to $C(Y)$, then $Y$ is also an almost discrete space.

(ii). Let $X$ be the one-point compactification of a discrete space and $C(X)$ be isomorphic with $C(Y)$. Then $Y$ is also the one-point compactification of a discrete space.

Proof. (i). If $X$ is an almost discrete space, then by Proposition 2.7, $\operatorname{Ann}\left(C_{F}(X)\right)=(0)$. Since the socle and its annihilator are stable under any isomorphism, $Y$ is also almost discrete by Proposition 2.7 . 
(ii). Let $\sigma: C(X) \rightarrow C(Y)$ be an isomorphism and $X$ be the one-point compactification of a discrete space with the nonisolated point $x_{\circ}$. Clearly for every $x_{\circ} \neq x \in X, O_{x}$ is generated by an idempotent. By Lemma 2.6, for every $x \in X, \sigma\left(O_{x}\right)=O^{y}$ for some $y \in \beta Y$. Then for $x \neq x_{\circ}, \sigma\left(O_{x}\right)=O^{y}$ is also generated by an idempotent; i.e., $y$ is an isolated point in $Y$. Now we suppose that $\sigma\left(O_{x_{\circ}}\right)=O^{y_{\circ}}$, where $y_{\circ} \in \beta Y$. If $y_{\circ} \notin Y$, then by Theorem 9.2 in [5], we have $|Y|=|\beta Y|=2^{2^{|Y|}}$, a contradiction. Therefore $y_{\circ} \in Y$. But $y_{\circ}$ is not an isolated point of $Y$, for otherwise $O^{y_{\circ}}=O_{y_{\circ}}$ is generated by an idempotent, for $C(X) \approx C(Y)$. This implies that $x_{\circ}$ is an isolated point, a contradiction. Therefore $y_{\circ}$ is the only nonisolated point of $Y$. Since the socle of $C(X)$ is invariant under $\sigma$ and since $C_{F}(X)=O_{x_{\circ}}$, it follows that $\sigma\left(C_{F}(X)\right)=O_{y_{\circ}}$; i.e., the socle of $C(Y)$ and $O_{y_{\circ}}$ coincide. Hence by Theorem $2.1, Y$ is the one-point compactification of a discrete space.

\section{Nowhere Compact and Almost Locally Compact Spaces}

The space $X$ is said to be nowhere compact, if every compact subset of $X$ has empty interior, and is said to be almost locally compact, if it has a dense locally compact subspace. For example, the space of rationals is a nowhere compact space. Clearly every locally compact space is almost locally compact, but not conversely. For example, a noncompact almost discrete space with only one nonisolated point is an almost locally compact but not locally compact. For another example, make the real numbers into a topological space by taking a base for the open sets consisting of the family of all open intervals and $\{\{r\}: r \in \mathbb{Q}\}$. For some topological properties of these spaces, see [9] and for an algebraic characterization of these spaces, see [2].

Clearly, every open subspace of an almost locally (nowhere) compact space is an almost locally (nowhere) compact space. In the preceding example, since $\mathbb{R} \backslash \mathbb{Q}$ is not almost locally compact, we conclude that the closed subspaces of an almost locally compact space need not be almost locally compact. Similarly, a singleton as a subspace of a nowhere compact space, evidently is not nowhere compact.

The proofs of the following propositions are trivial.

Proposition 3.1. $X$ is an almost locally compact space if and only if every nonempty open subset of $X$ contains an open set with compact closure.

Proposition 3.2. (i). The free union $\stackrel{\circ}{\cup}_{s \in S} X_{s}$ is an almost locally (nowhere) compact space if and only if each $X_{s}$ is.

(ii). The cartesian product $\prod_{s \in S} X_{s}$ is a nowhere compact space if and only if each $X_{s}$ is. 
(iii). The cartesian product $\prod_{s \in S} X_{s}$, where $X_{s} \neq \phi, \forall s \in S$, is an almost locally compact space if and only if all spaces $X_{s}$ are almost locally compact and there exists a finite set $S_{0} \subseteq S$ such that $X_{s}$ is compact for every $s \in S \backslash S_{0}$.

The following Theorem shows that $C_{\infty}(\mathbb{Q})=C_{K}(\mathbb{Q})=(0)$, (see also $4 \mathrm{D}$ of $[5])$.

Theorem 3.3. The following statements are equivalent:

(i) $X$ is nowhere compact.

(ii) $C_{\infty}(X)=(0)$.

(iii) $C_{K}(X)=(0)$.

Proof. $(i) \Rightarrow(i i)$. Let $X$ be a nowhere compact space and $f \in C_{\infty}(X)$. Then the set $\left\{x:|f(x)| \geq \frac{1}{n}\right\}$ is compact for every $n$ and by our hypothesis, $\operatorname{int}\left\{x:|f(x)| \geq \frac{1}{n}\right\}=\phi$. But for every $n>1$,

$$
\left\{x:|f(x)|>\frac{1}{n-1}\right\} \subseteq \operatorname{int}\left\{x:|f(x)| \geq \frac{1}{n}\right\}=\phi
$$

implies that $f=0$; i.e., $C_{\infty}(X)=(0)$.

$($ ii $) \Rightarrow($ iii $)$. The proof is clear, for $C_{K}(X) \subseteq C_{\infty}(X)$.

$($ iii $) \Rightarrow(i)$. Suppose that $C_{K}(X)=(0)$ and $A$ is a compact subset of $X$. If int $A \neq \phi$, we consider $x \in \operatorname{int} A$ and define $f \in C(X)$ such that $f(x)=1$ and $f(X \backslash \operatorname{int} A)=\{0\}$. Since $\operatorname{cl}(X \backslash Z(f)) \subseteq A$, it follows that $\operatorname{cl}(X \backslash Z(f))$ is also compact; so $f \in C_{K}(X)=(0)$ implies that $f=0$, a contradiction.

Corollary 3.4. $X$ is a nowhere compact space if and only if $\beta X \backslash X$ is dense in $\beta X$.

Proof. By our Theorem 3.3 and 7F in [5], the proof is evident.

It is well known that $\cap Z\left[C_{K}(X)\right]=\phi$ if and only if $X$ is locally compact but not compact (see 4D in [5]). It is also proved in [2], Theorem 3.2, that $X$ is almost locally compact if and only if int $\cap Z\left[C_{K}(X)\right]=\phi$; i.e., if and only if $C_{K}(X)$ is an essential ideal. The following Theorem generalizes and unifies these results.

Theorem 3.5. If $\cap Z\left[C_{K}(X)\right]=Y$, then $X \backslash Y$ is an open locally compact subspace of $X$ and $\operatorname{int}_{X} Y$ is a nowhere compact subspace of $X$. Conversely, if $Y \subseteq X$ and $X \backslash Y$ is an open locally compact subspace of $X$, then $\cap Z\left[C_{K}(X)\right] \subseteq$ $Y$. 
Proof. Let $\cap Z\left[C_{K}(X)\right]=Y$ and $x \in X \backslash Y$. Then there exists $f \in C_{K}(X)$ such that $x \in X \backslash Z(f)$. Since $G=(X \backslash Z(f)) \cap(X \backslash Y)$ is an open set in $X$ containing $x$, by the regularity of $X$, there exists an open set $V$ in $X$ such that $x \in V \subseteq \operatorname{cl} V \subseteq G \subseteq \operatorname{cl}(X \backslash Z(f))$. But $f \in C_{K}(X)$; i.e., $\operatorname{cl}(X \backslash Z(f))$ is compact and hence $\mathrm{cl} V$ is a compact neighborhood of $x$ in $X$. Since $X \backslash Y$ is an open subspace of $X$ and $\operatorname{cl} V \subseteq X \backslash Y$, it follows that $\operatorname{cl} V$ is also a compact neighborhood of $x$ in $X \backslash Y$; i.e., $X \backslash Y$ is locally compact. To prove the nowhere compactness of int $Y$, let $x \in \operatorname{int} Y$ and $G \neq \phi$ be a compact neighborhood of $x \operatorname{in} \operatorname{int} Y$. Since int $Y$ is an open subspace of $X, G$ is also a compact neighborhood of $x$ in $X$. By the regularity of $X$ and Theorem 1.15 in [5], there exists $f \in C(X)$ such that $x \in X \backslash Z(f) \subseteq \operatorname{cl}(X \backslash Z(f)) \subseteq G$. But $G$ is compact in $X$; so $\operatorname{cl}(X \backslash Z(f))$ is also compact in $X$; i.e., $f \in C_{K}(X)$ implies that $x \notin \cap Z\left[C_{K}(X)\right]$, a contradiction. Therefore int $Y$ is nowhere compact.

Conversely, let $X \backslash Y$ be an open locally compact subspace of $X$. If $x \in$ $\cap Z\left[C_{K}(X)\right]$ and $x \notin Y$, then $x$ has a compact neighborhood $G$ in $X \backslash Y$, for $X \backslash Y$ is locally compact. Since $X \backslash Y$ is open in $X, G$ is also a compact neighborhood in $X$. Again by Theorem 1.15 in [5], there exists $f \in C(X)$ such that $x \in X \backslash Z(f) \subseteq \operatorname{cl}(X \backslash Z(f)) \subseteq G$. The compactness of $G$ implies that $\operatorname{cl}(X \backslash Z(f))$ is also compact in $X$; i.e., $f \in C_{K}(X)$, which contradicts $x \in \cap Z\left[C_{K}(X)\right]$. Hence $x \in Y$; i.e., $\cap Z\left[C_{K}(X)\right] \subseteq Y$.

Corollary 3.6. Every completely regular space $X$ is the union of two disjoint subspaces $A$ and $N$, where $A$ is closed almost locally compact and $N$ is open nowhere compact.

Proof. By Theorem 3.5, we can consider $A=X \backslash \operatorname{int} Y$ and $N=\operatorname{int} Y$, where $Y=\cap Z\left[C_{K}(X)\right]$. Since $X \backslash Y$ is locally compact and $\operatorname{cl}(X \backslash Y)=X \backslash \operatorname{int} Y$, it follows that $A$ is a closed almost locally compact subspace of $X$. Clearly int $Y$ is an open nowhere compact subspace of $X$ by Theorem 3.5. Moreover $X=A \cup N$.

Corollary 3.7. $X$ is almost locally compact if and only if $C_{K}(X)$ is essential. (See also [2], Theorem 3.2).

Proof. If $X$ is almost locally compact, then by Theorem 3.5 , int $Y=\phi$, where $\cap Z\left[C_{K}(X)\right]=Y$. Therefore $C_{K}(X)$ is essential by Proposition 1.1

Conversely, whenever $C_{K}(X)$ is essential, then by Proposition 1.1, int $Y=$ $\phi$, where $Y=\cap Z\left[C_{K}(X)\right]$. Therefore $X$ is almost locally compact by Theorem 3.5 .

Corollary 3.8. $C_{K}(X)$ is free if and only if $X$ is locally compact but not compact. (See also [5], 4D.3). 
Corollary 3.9. $C_{K}(X)$ is generated by an idempotent if and only if $X$ is the union of two disjoint open subspaces $C$ and $N$, where $C$ is compact and $N$ is nowhere compact.

Proof. Let $C_{K}(X)=(e)$, where $e$ is an idempotent. Then $Y=\cap Z\left[C_{K}(X)\right]=$ $Z(e)$ is an open set and hence int $Y=Y$. By Theorem 3.5, if we consider $C=X \backslash Z(e)$ and $N=Z(e)$, then $N$ is open nowhere compact and $e \in C_{K}(X)$ implies that $\operatorname{cl}(X \backslash Z(e))=X \backslash Z(e)=C$ is an open compact subspace of $X$. Conversely, suppose that $X=C \cup N$, where $C$ and $N$ are two disjoint open subspaces of $X, C$ is compact and $N$ is nowhere compact. Define the idempotent $e \in C_{K}(X)$ such that $e(N)=\{0\}$ and $e(C)=\{1\}$, we will show that $C_{K}(X)=(e)$. Since $X \backslash Z(e)=C$ is compact, $e \in C_{K}(X)$. Now if $f \in C_{K}(X)$, then $\operatorname{cl}(X \backslash Z(f)$ is compact and clearly $\operatorname{cl}(X \backslash Z(f)) \cap N$ is also a compact subset of $N$; hence it must have empty interior. But $(X \backslash Z(f)) \cap N \subseteq \operatorname{int}(\operatorname{cl}(X \backslash Z(f)) \cap N)$ implies that $(X \backslash Z(f)) \cap N=\phi$. Therefore $(X \backslash Z(f)) \subseteq C=X \backslash Z(e)$; i.e., $Z(e) \subseteq \operatorname{int} Z(f)$ implies that $f$ is a multiple of $e$ by $1 \mathrm{D}$ in [5]; i.e., $C_{K}(X)=(e)$.

\section{Pseudocompact and Lindelöf Spaces}

An ideal $I$ in a commutative ring $R$ is said to be divisible if for every two members $a$ and $b$ in $I$ there exists $c \in I$ such that $a$ and $b$ are multiples of $c$. For example, every principal ideal is a divisible ideal. It is also shown in [7] that any ideal in $C(X)$ which is an intersection of prime ideals is a divisible ideal. This fact and Theorem 4.11 in [5] imply that $X$ is compact if and only if every divisible ideal in $C(X)$ is fixed.

A strongly divisible ideal in a commutative ring $R$ is an ideal $I$ which satisfies the following condition: $a_{1}, a_{2}, \ldots$ in $I$ implies the existence of elements $e \in I$ and $b_{1}, b_{2}, \ldots$ in $R$ such that $a_{i}=e b_{i}$, for $i=1,2, \ldots$. Clearly every strongly divisible ideal is a divisible ideal but not conversely. It is shown in Corollary 4.3 that every real maximal ideal (see [5], for definition) is a strongly divisible ideal. Clearly a countably generated ideal is strongly divisible if and only if it is a principal ideal. In this section we will show that $X$ is Lindelöf if and only if every strongly divisible ideal of $C(X)$ is fixed.

To prove the main results of this section we need the following lemma.

Lemma 4.1. If $I$ is a z-ideal and $Z[I]$ is closed under countable intersection, then $I$ is a strongly divisible ideal. 
Proof. Let $f_{n} \in I$. Since $I$ is a z-ideal, then $f_{n}^{2 / 3} \in I, \forall n \in \mathbb{N}$. Put

$$
g=\sum_{n=1}^{\infty} \frac{1}{2^{n}} \frac{f_{n}^{2 / 3}}{1+f_{n}^{2 / 3}}
$$

Because of uniform convergence, $g \in C(X)$. But $Z(g)=\cap_{n=1}^{\infty} Z\left(f_{n}\right)$ and $Z[I]$ is closed under countable intersection. Hence $Z(g) \in Z[I]$ implies that $g \in I$, for $I$ is a z-ideal. By definition of $g$, we have $\frac{\left|f_{n}\right|}{\left(1+f_{n}^{\frac{2}{3}}\right)^{\frac{3}{2}}} \leq 2^{n}|g|^{3 / 2}$ and 1D in [5] implies that $f_{n}$ is a multiple of $g, \forall n \in \mathbb{N}$; i.e., $I$ is a strongly divisible ideal.

Corollary 4.2. Every real maximal ideal is a strongly divisible ideal.

PRoOF. Using 5.14 in [5] and Lemma 4.1, the proof is evident.

Corollary 4.3. $X$ is pseudocompact if and only if every ideal is contained in a strongly divisible z-ideal.

Proof. By 5H in [5] and Lemma 4.1, the proof is evident.

Theorem 4.4. $X$ is Lindelöf if and only if every strongly divisible ideal in $C(X)$ is fixed.

Proof. First suppose that $X$ is Lindelöf and $I$ is a strongly divisible ideal in $C(X)$. Then $Z[I]$ has the countable intersection property and hence $Z[I]$ is fixed; i.e., $I$ is fixed.

Conversely, let every strongly divisible ideal be fixed and $\mathbb{A}$ be a collection of closed sets in $X$ which is closed under countable intersection. If we denote by $\mathbb{A}_{z}$ the collection of all zero-sets containing a member of $\mathbb{A}$, then clearly the collection $\mathfrak{F}_{\circ}$ containing $\mathbb{A}_{z}$ and all countable intersections of members of $\mathbb{A}_{z}$ is a z-filter base for a z-filter $\mathfrak{F}$. Therefore $Z^{-1}(\mathfrak{F})=\{f \in C(X): Z(f) \in \mathfrak{F}\}$ is a $z$-ideal and $\mathfrak{F}$ is closed under countable intersection. By Lemma 4.1, $Z^{-1}(\mathfrak{F})$ is strongly divisible and hence it is fixed by our hypothesis. Then $\cap_{B \in \mathbb{A}} B \supseteq \cap_{Z \in \mathbb{A}_{z}} Z \supseteq \cap_{Z \in \mathfrak{F}} Z \neq \phi$ implies that $X$ is Lindelöf.

\section{References}

[1] F. Azarpanah, Essential ideals in $C(X)$, Priod. Math. Hungar., 31(2) (1995), 105-112.

[2] -, Intersection of essential ideals in $C(X)$, Proc. Amer. Math. Soc., 125(7) (1997),2149-2154. 
[3] W. E. Dietrich, Jr., On the ideal structure of $C(X)$, Trans. Amer. Math. Soc., 152 (1970), 61-77.

[4] R. Engelking, General topology, PWN Polish Scientific Publishers, 1977.

[5] L. Gillman and M. Jerison, Rings of continuous functions, SpringerVerlag, 1976.

[6] K. R. Goodearl, Von Newmann regular rings, Pitman, San Francisco, 1979.

[7] J. G. Horne, On $O_{w}$-ideals in $C(X)$, Proc. Amer. Math. Soc., 9 (1958), $511-518$.

[8] O. A. S. Karamzadeh and M. Rostami, On the intrinsic topology and some related ideals of $C(X)$, Proc. Amer. Math. Soc., 93 (1985), 179-184.

[9] M. C. Rayburn, Compactifications with almost locally compact outgrowth, Proc. Amer. Math. Soc., 106 (1984), 223-229.

[10] S. Willard, General topology, Addison Wesley, Reading Mass., 1970. 
F. Azarpanah 